\title{
Wide-Angle Propagating Beam Analysis for Circularly Symmetric Waveguides: Comparison Between FD-BPM and FD-TDM
}

\author{
Junji Yamauchi, Member, IEEE, Yasuhiro Akimoto, Masayuki Nibe, and Hisamatsu Nakano, Fellow, IEEE
}

\begin{abstract}
The finite-difference beam-propagation method (FDBPM) with a $(1,1)$ Padé approximant operator is formulated for circularly symmetric waveguides. A tapered step-index fiber is analyzed, and the computed field distribution is compared with that from the scalar finite-difference time-domain method (FDTDM). The present method offers improvement in the evaluation of a radiation-mode field compared with the conventional FDBPM based on the paraxial approximation.
\end{abstract}

\section{INTRODUCTION}

T VHE finite-difference beam-propagation method [1] (FDBPM) is an efficient means of analyzing the propagating beam in an optical waveguide. However, it is well-known in the rectangular coordinates that the accuracy of the FD-BPM is degraded in the analysis of wide-angle propagation because the paraxial approximation is employed. To avoid this degradation, several techniques have been proposed [2]-[3] using Padé approximant operators. Recently, Yevick and Hermansson [4] discussed the convergence properties of wide-angle techniques.

Since the conventional BPM in the rectangular coordinates results in reduced computational efficiency for propagation problems in circularly symmetric waveguides, a method of analyzing a propagating field in the cylindrical coordinates has also received much attention [5]-[9]. All the BPM's in the cylindrical coordinates reported so far are based on the paraxial approximation. It should be noted that the BPM based on the finite-difference techniques [9] has the advantage that a transparent boundary condition [10] is easily incorporated. However, it can be predicted that the accuracy of the conventional FD-BPM for circularly symmetric waveguides is also degraded in the analysis of wide-angle propagation.

In this letter, we formulate a FD-BPM with a $(1,1)$ Padé approximant operator for circularly symmetric waveguides to attain a high degree of accuracy in analyzing wide-angle beam propagation. We treat a tapered step-index fiber, and compare the field distribution computed from the present method with that from the scalar finite-difference time-domain method (FD-TDM) [11] in the cylindrical coordinates [12]. The FDTDM has penalty in the computation time, but the result is accurate since no approximation is introduced with respect to the propagating beam direction. Recently, some discrepancy

Manuscript received August 25, 1995; revised October 24, 1995.

The authors are with the College of Engineering, Hosei University, 3-7-2, Kajino-cho, Koganei, Tokyo 184, Japan.

Publisher Item Identifier S 1041-1135(96)00947-0. between the FD-TDM and the FD-BPM based on the paraxial approximation was reported in the evaluation of a radiationmode field in a slab optical waveguide [13]. This is partly due to the fact that a radiation-mode field includes wideangle propagating beam components. Therefore, we check the accuracy in the wide-angle beam propagation using the results obtained from the FD-TDM. We show that the present method offers improvement in the analysis of a radiation-mode field, while maintaining the advantages of the conventional FD-BPM.

\section{FORMULATION}

We consider the propagation of a field of $E(r, \phi, z)$ in an optical fiber. The scalar Helmholtz equation in the cylindrical coordinates is

$$
\frac{\partial^{2} E}{\partial z^{2}}+\frac{\partial^{2} E}{\partial r^{2}}+\frac{1}{r} \frac{\partial E}{\partial r}+\frac{1}{r^{2}} \frac{\partial^{2} E}{\partial \phi^{2}}+k_{0}^{2} n^{2}(r, \phi, z) E=0
$$

where $k_{0}$ is the wavenumber in free space, $E(r, \phi, z) \exp (j \omega t)$ is a component of the electric field at an angular frequency of $\omega$, and $n(r, \phi, z)$ is the index profile of the waveguide. For convenience, the electric field $E$ is expressed as

$$
E(r, \phi, z)=\psi(r, z) \exp \left(-j k_{0} n_{0} z\right) \exp (j l \phi)
$$

where $n_{0}$ is a reference refractive index and $l$ is integer. Substituting (2) into (1), we obtain

$$
-\frac{\partial^{2} \psi}{\partial z^{2}}+2 j k_{0} n_{0} \frac{\partial \psi}{\partial z}=\left\{\frac{\partial^{2}}{\partial r^{2}}+\frac{1}{r} \frac{\partial}{\partial r}-\frac{l^{2}}{r^{2}}+k_{0}^{2}\left(n^{2}-n_{0}^{2}\right)\right\} \psi
$$

(3) reduces to the paraxial equation, when we neglect the term of $\frac{\partial^{2}}{\partial z^{2}}$. Here, $\frac{\partial^{2}}{\partial z^{2}}$ is evaluated by the following way [3].

We may formally rewrite (3) in the form:

$$
\frac{\partial \psi}{\partial z}=-\frac{\frac{j P}{2 k_{0} n_{0}}}{1+\frac{j}{2 k_{0} n_{0}} \frac{\partial}{\partial z}} \psi
$$

where

$$
P=\frac{\partial^{2}}{\partial r^{2}}+\frac{1}{r} \frac{\partial}{\partial r}-\frac{l^{2}}{r^{2}}+k_{0}^{2}\left(n^{2}-n_{0}^{2}\right)
$$

When the recurrence relation with respect to $\frac{\partial}{\partial z}$ is utilized, the following simplified equation with the $(1,1)$ Padé operator 
is obtained:

$$
\frac{\partial \psi}{\partial z}=-\frac{\frac{j P}{2 k_{0} n_{0}}}{1+\frac{P}{4 k_{0}^{2} n_{0}^{2}}} \psi .
$$

To (6), the Crank-Nicholson (CN) scheme is applied and the difference equation is derived. The $\mathrm{CN}$ scheme is an implicit method and is known to be highly stable. For the difference equations, the following simplifying symbol will be used:

$$
\psi(r, z)=\psi((i-1) \Delta r, k \Delta z)=\psi_{i}^{k}
$$

where $i$ and $k$ are integers. Then the difference equation for $i>1$ is as follows:

$$
\begin{aligned}
a^{+} \psi_{i-1}^{k+1} & +\left(\frac{4 k_{0}^{2} n_{0}^{2} \Delta r^{2}}{1+j k_{0} n_{0} \Delta z}+\zeta\right) \psi_{i}^{k+1}+a^{-} \psi_{i+1}^{k+1} \\
=\xi a^{+} \psi_{i-1}^{k} & +\left(\frac{4 k_{0}^{2} n_{0}^{2} \Delta r^{2}}{1+j k_{0} n_{0} \Delta z}+\xi \zeta\right) \psi_{i}^{k}+\xi a^{-} \psi_{i+1}^{k}
\end{aligned}
$$

where $a^{ \pm}=1 \mp \frac{1}{2(i-1)}, \zeta=\Delta r^{2} k_{0}^{2}\left(n^{2}-n_{0}^{2}\right)-\Delta r^{2} \frac{l^{2}}{r^{2}}-2$ and

$$
\xi=\frac{1-j k_{0} n_{0} \Delta z}{1+j k_{0} n_{0} \Delta z}
$$

The indeterminate form at the origin $r=0$ can be evaluated by L'Hospital's rule. Taking into account the circular symmetry of the field, $\psi_{0}^{k}=\psi_{2}^{k} \exp (j l \pi)$, we obtain for $i=1$ :

$$
\begin{aligned}
\left(\frac{4 k_{0}^{2} n_{0}^{2} \Delta r^{2}}{1+j k_{0} n_{0} \Delta z}+\eta\right) \psi_{1}^{k+1}+b \psi_{2}^{k+1} & \\
& =\left(\frac{4 k_{0}^{2} n_{0}^{2} \Delta r^{2}}{1+j k_{0} n_{0} \Delta z}+\xi \eta\right) \psi_{1}^{k}+\xi b \psi_{2}^{k}
\end{aligned}
$$

where

$$
b=\left(1+\exp ^{j l \pi}\right)\left(2-\frac{l^{2}}{2}\right)
$$

and

$$
\eta=\Delta r^{2} k_{0}^{2}\left(n^{2}-n_{0}^{2}\right)-2\left(2-\frac{l^{2}}{2}\right) .
$$

(8) and (9) lead to a tridiagonal system of linear equations. Hence, an efficient computing algorithm can be used as in the case of the FD-BPM with the paraxial approximation. It should also be noted that a transparent boundary condition [10] can easily be incorporated in the present method.

Next, we formulate the scalar FD-TDM for circularly symmetric waveguides [12]. The formulation can be regarded as an extension of the scalar FD-TDM in the rectangular coordinates [11] to the cylindrical coordinates. We assume that $n$ is independent of time. The electric field can be expressed as

$$
E(r, \phi, z, t)=\psi(r, z, t) \exp (j l \phi)
$$

so that a scalar wave equation becomes

$$
\frac{\partial^{2} \psi}{\partial r^{2}}+\frac{1}{r} \frac{\partial \psi}{\partial r}-\frac{l^{2}}{r^{2}} \psi+\frac{\partial^{2} \psi}{\partial z^{2}}-\frac{n^{2}}{c^{2}} \frac{\partial^{2} \psi}{\partial t^{2}}=0
$$

where $c$ is the velocity of light in free space. Using a central difference formula, we derive

$$
\begin{aligned}
\psi_{i}^{k}(m+1)= & 2\left[1-\left\{1+\frac{1}{2}\left(\frac{l}{i-1}\right)^{2}\right\}\left(\frac{c \Delta t}{n \Delta r}\right)^{2}\right. \\
& \left.-\left(\frac{c \Delta t}{n \Delta z}\right)^{2}\right] \psi_{i}^{k}(m)-\psi_{i}^{k}(m-1) \\
& +\left(\frac{c \Delta t}{n \Delta r}\right)^{2}\left[\left\{1+\frac{1}{2(i-1)}\right\} \psi_{i+1}^{k}(m)\right. \\
& \left.+\left\{1-\frac{1}{2(i-1)}\right\} \psi_{i-1}^{k}(m)\right] \\
& +\left(\frac{c \Delta t}{n \Delta z}\right)^{2}\left[\psi_{i}^{k+1}(m)+\psi_{i}^{k-1}(m)\right]
\end{aligned}
$$

where $n=n_{i}^{k}, t=(m-1) \Delta t, r=(i-1) \Delta r$, and $z=(k-$ 1) $\Delta z$, in which $\Delta t, \Delta r$ and $\Delta z$ represent increments along the time, transverse and propagation directions, respectively.

On the fiber axis, $r=0$, we again apply L'Hospital's rule to the second and third terms of (11). Then, we have

$$
\left(2-\frac{l^{2}}{2}\right) \frac{\partial^{2} \psi}{\partial r^{2}}+\frac{\partial^{2} \psi}{\partial z^{2}}-\frac{n^{2}}{c^{2}} \frac{\partial^{2} \psi}{\partial t^{2}}=0 .
$$

(13) reduces to the following difference equation:

$$
\begin{aligned}
\psi_{1}^{k}(m+1)= & 2\left[1-\left(2-\frac{l^{2}}{2}\right)\left(\frac{c \Delta t}{n \Delta r}\right)^{2}\right. \\
& \left.-\left(\frac{c \Delta t}{n \Delta z}\right)^{2}\right] \psi_{1}^{k}(m)-\psi_{1}^{k}(m-1) \\
& +(1+\exp (j l \pi))\left(2-\frac{l^{2}}{2}\right)\left(\frac{c \Delta t}{n \Delta r}\right)^{2} \psi_{2}^{k}(m) \\
& +\left(\frac{c \Delta t}{n \Delta z}\right)^{2}\left[\psi_{1}^{k+1}(m)+\psi_{1}^{k-1}(m)\right]
\end{aligned}
$$

where $n=n_{1}^{k}$. In contrast to the FD-BPM, the FD-TDM is an explict scheme in which the field values at two previous time steps are required. At the edge of the calculation region, we apply the Mur's first-order absorbing boundary condition [14].

\section{RESULTS}

We analyze a tapered step-index fiber shown in the inset of Fig. 1. The refractive indices of the core and cladding are $n_{c o}=1.5075$ and $n_{c l}=1.5$, respectively. The initial core radius is $r_{\mathrm{c}}=5.167 \mu \mathrm{m}$, and the wavelength of $\lambda=1.55 \mu \mathrm{m}$ is used, so that the normalized frequency of the input fiber is taken to be $V \cong 3.15$. The input field is the second-order mode $L P_{11}$, which can be obtained analytically. The fiber is tapered with $\theta_{t}=15.0^{\circ}$ and $L_{t}=15.5 \mu \mathrm{m}$, and is connected with a single-mode fiber. The computation parameters are taken to be $\Delta r=r_{c} / 199.5 \cong 0.0259 \mu \mathrm{m}$ and $\Delta z=0.0250 \mu \mathrm{m}$. The number of transverse grid points is 500 . We compare the field distribution computed from the FD-BPM with that from the FD-TDM $(c \Delta t=0.0020 \mu \mathrm{m})$. The discretization mesh for each method is taken to be the same. For the analysis using the FD-TDM, we employ a monochromatic driving light source that generates the $L P_{11}$ mode at the entrance of the taper. The 


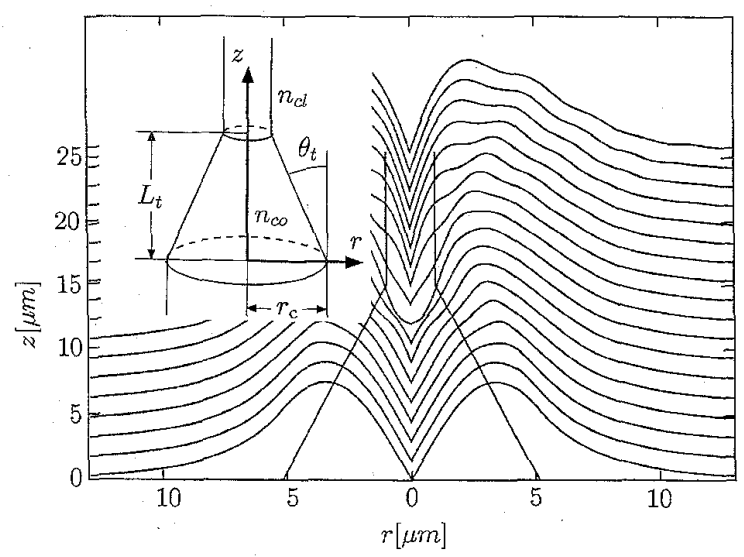

Fig. 1. Geometry of a tapered step-index fiber and its typical field distribution obtained from the FD-BPM with a $(1,1)$ Padé approximant operator.

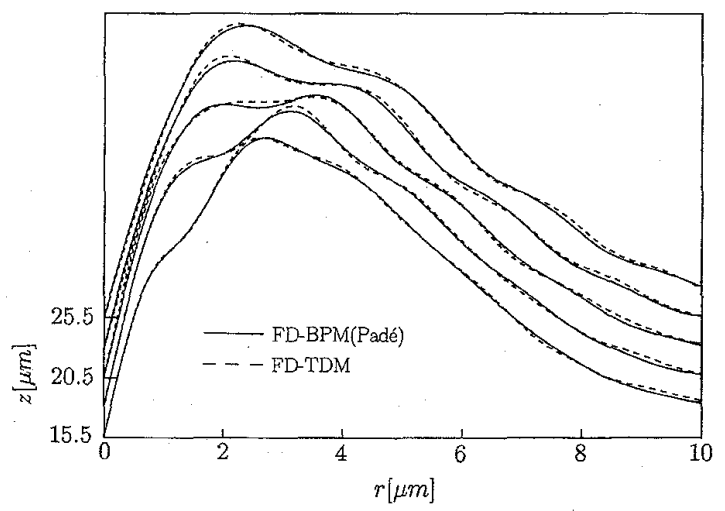

(a)

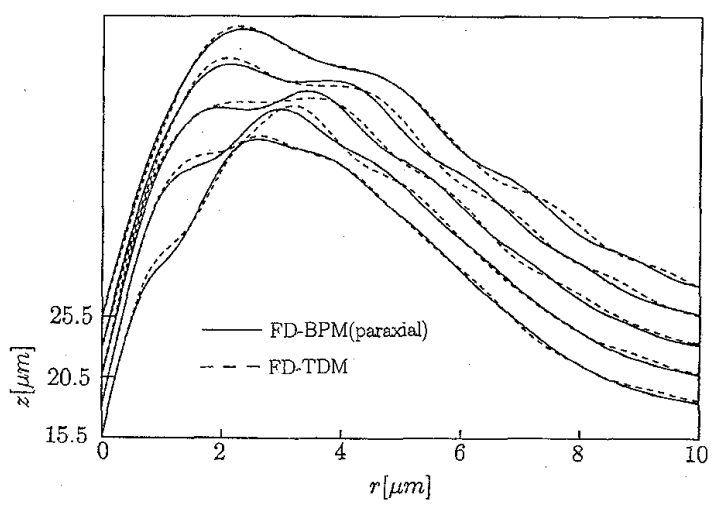

(b)

Fig. 2. Comparison between the propagating fields obtained from the FD-BPM and the FD-TDM.

resulting field is propagated in time until a steady state field is obtained.

A typical field distribution obtained from the FD-BPM along the tapered fiber is shown in Fig. 1. To compare the fields obtained from different techniques in detail, we only show the propagating field between $z=15.5 \mu \mathrm{m}$ and $25.5 \mu \mathrm{m}$ in Fig. 2. The field obtained from the FD-TDM is that observed at $c t=100 \mu \mathrm{m}$ (The field almost reaches a steady state at $c t=50 \mu \mathrm{m}$ ). For comparison, the results obtained from the FD-BPM using the paraxial approximation are also shown in Fig. 2(b). Due to tapering, the $L P_{11}$ mode is cut off. Therefore, the radiation-mode field is generated and is spread as it propagates. It is found that using the paraxial approximation makes an error in wide-angle propagation. For the FD-BPM using the $(1,1)$ Padé approximant operator, the field distribution fairly agrees with that computed from the FD-TDM.

\section{CONCLUSION}

Finite-difference beam-propagation method with a $(1,1)$ Padé approximant operator for circularly symmetric waveguides has been formulated to improve the accuracy in wideangle beam propagation. For comparison, we have also formulated the scalar FD-TDM in the cylindrical coordinates. The analysis of a tapered fiber shows that the present method has reasonable accuracy in the evaluation of a radiation-mode field, while maintaining the advantages of a FD-BPM based on the paraxial approximation.

\section{REFERENCES}

[1] Y. Chung and N. Dagli, "An assessment of finite difference beam propagation method," IEEE J. Quantum Electron., vol. 26, no. 8, pp. 1335-1339, 1990.

[2] D. Yevick and M. Glasner, "Forward wide-angle light propagation in semiconductor rib waveguides," Opt. Lett., vol. 15, no. 3, pp. 174-176, 1990.

[3] G.R.Hadley, "Wide-angle beam propagation using Pade approximant operators," Opt. Lett., vol. 17, no. 20, pp. 1426-1428, 1992.

[4] D. Yevick and B. Hermansson, "Convergence properties of wide-angle techniques," IEEE Photon. Technol. Lett., vol. 6, no. 12, pp. 1457-1459, 1994.

[5] M. D. Feit and J. A. Fleck, Jr., "Simple spectral method for solving propagation problems in cylindrical geometry with fast Fourier transforms," Opt. Lett., vol. 14, no. 13, pp. 662-664, 1989.

[6] K. Hayata, A. Misawa, and M. Koshiba, "Split-step finite-element method applied to nonlinear integrated optics," J. Opt. Soc. Am. B, vol. 7, no. 9, pp. 1772-1784, 1990.

[7] F. Gonthier, A. Hénault, S. Lacroix, R. J. Black, and J. Bures, "Mode coupling in nonuniform fibers: comparison between coupled-mode theory and finite-difference beam-propagation method simulations," J. Opt. Soc. Am. B, vol. 8, no. 2, pp. 416-421, 1991.

[8] M. Kuroda, A. Maruta, and M. Matsuhara, "Analysis of lightwave propagation in an axisymmetric optical circuit by the Galerkin method," IEE Japan, Tech. Meeting, EMT-92-38, pp. 11-19, 1992.

[9] J. Yamauchi, M. Ikegaya, T. Ando, and H. Nakano, "Finite-difference beam-propagation method for circularly symmetric fields, "IEICE Trans. Electron., vol. E75-C, no. 9, pp. 1093-1095, 1992.

[10] G. R. Hadley, "Transparent boundary condition for beam propagation," Opt. Lett., vol. 16, no. 9, pp. 624-626, 1991.

[11] W. P. Huang, S. T. Chu, A. Goss, and S. K. Chaudhuri, "A scalar finitedifference time-domain approach to guided-wave optics," IEEE Photon. Technol. Lett., vol. 3, no. 6, pp. 524-526, 1991.

[12] J. Yamauchi, M. Nibe, and H. Nakano, "A scalar FD-TD method for circularly symmetric fields," IEICE Nat. Conv. Rec., C-133, 1994.

[13] Y. Naka, H. Ikuno, A.Yata, and M. Nishimoto, "Analysis of waveguide discontinuity using the FD-TD method," IEICE Nat. Conv. Rec., C-361, 1995.

[14] G. Mur, "Absorbing boundary conditions for the finite-difference approximation of the time-domain electromagnetic-field equations," IEEE Trans. Electromag. Compat., vol. EMC-23, no. 4, pp. 377-382, 1981. 